\title{
Obesity occurring in apolipoprotein E-knockout mice has mild effects on fertility
}

\author{
Ting Zhang*, Pengyuan Dai ${ }^{1, *}$, Dong Cheng ${ }^{2}$, Liang Zhang ${ }^{1}$, Zijiang Chen, Xiaoqian Meng ${ }^{1}$, \\ Fumiao Zhang ${ }^{1}$, Xiaoying Han ${ }^{1}$, Jianwei Liu ${ }^{1}$, Jie Pan ${ }^{1}$, Guiwen Yang ${ }^{1}$ and Cong Zhang \\ Shanghai Key Laboratory for Assisted Reproduction and Reproductive Genetics, Renji hospital, Shanghai Jiao Tong \\ University School of Medicine, Shanghai 200135, China, ${ }^{1}$ Key Laboratory of Animal Resistance Research, College of \\ Life Science, Shandong Normal University, 88 East Wenhua Road, Ji'nan, Shandong 250014, China and \\ ${ }^{2}$ Shandong Center for Disease Control and Prevention, 16992 Jingshi Road, Ji'nan, Shandong 250014, China \\ Correspondence should be addressed to C Zhang; Email: zhangxinyunlife@163.com
}

*(T Zhang and P Dai contributed equally to this work)

\begin{abstract}
The Apolipoprotein (Apo) family is implicated in lipid metabolism. There are five types of Apo: Apoa, Apob, Apoc, Apod, and Apoe. Apoe has been demonstrated to play a central role in lipoprotein metabolism and to be essential for efficient receptor-mediated plasma clearance of chylomicron remnants and VLDL remnant particles by the liver. Apoe-deficient $\left(\right.$ Apoe $^{-/-}$) mice develop atherosclerotic plaques spontaneously, followed by obesity. In this study, we investigated whether lipid deposition caused by Apoe knockout affects reproduction in female mice. The results demonstrated that $A_{p o e}{ }^{-/-}$mice were severely hypercholesterolemic, with their cholesterol metabolism disordered, and lipid accumulating in the ovaries causing the ovaries to be heavier compared with the WT counterparts. In addition, estrogen and progesterone decreased significantly at D 100 . Quantitative PCR analysis demonstrated that at D 100 the expression of cytochromeP450 aromatase (Cyp19a1), 3ß-hydroxysteroid dehydrogenase (Hsd3b), mechanistic target of rapamycin (Mtor), and nuclear factor-КB (Nfkb) decreased significantly, while that of BCL2-associated agonist of cell death (Bad) and tuberous sclerosis complex 2 (Tsc2) increased significantly in the Apoe $^{-/-}$mice. However, there was no difference in the fertility rates of the Apoe $^{-/-}$and WT mice; that is, obesity induced by Apoe knockout has no significant effect on reproduction. However, the deletion of Apoe increased the number of ovarian follicles and the ratio of ovarian follicle atresia and apoptosis. We believe that this work will augment our understanding of the role of Apoe in reproduction.

Reproduction (2014) 147 141-151
\end{abstract}

\section{Introduction}

Obesity has grown to epidemic proportions, and currently nearly half of reproductive age women are overweight or obese. Obesity has been demonstrated consistently to have a detrimental effect on the female reproductive system (Devlieger et al. 2008, Ogbuji 2010). Obesity and overweight are correlated with endocrine disorders as well as polycystic ovary syndrome in women; moreover, obesity affects ovulation and pregnancy rates, increases infertility risk, and causes irregular periods, miscarriage, birth defects, and even stillbirth (Bellver et al. 2007). Female reproductive success depends on the recruitment and development of ovarian follicles, oocyte maturation, ovulation, fertilization, and pregnancy. The whole process is regulated by steroid sex hormones such as estrogen, progesterone, and the gonadotropins follicle-stimulating hormone $(\mathrm{FSH})$ and luteinizing hormone (LH) through endocrine, autocrine, and paracrine mechanisms coordinately (Hirshfield 1991, Dasgupta et al. 2012). These sterol hormones have a close relationship with cholesterol metabolism because cholesterol functions as a substrate for hormone biosynthesis. Although there are three immediate sources of cholesterol for steroidogenic cells: de novo synthesis, lipoprotein cholesterol, and intracellular cholesteryl ester stores, studies employing oral or i.v. administration of labeled cholesterol and cholesterol precursors such as acetate have indicated that as much as $80 \%$ or more of the steroidogenic substrate of the adrenals, corpora lutea $(\mathrm{CL})$, and placentas arises from extracellular sources (Gwynne \& Strauss 1982).

Apolipoprotein E (APOE) is an arginine-rich Apo with a relative molecular mass of $34000 \mathrm{Da}$ and was first 
identified as a lipoprotein constituent of VLDLs by Shore \& Shore (1973). APOE is recognized as an important molecule serving various functions, including lipoprotein structure maintenance, lipoprotein metabolism regulation, and immunoregulation as well as neuronal repair. The attention paid to the role of Apoe in lipoprotein metabolism has been generated from the observation of APOE-enriched VLDLs accumulated in the plasma of patients with type III hyperlipoproteinemia, a genetic disorder (Mahley 1988). Furthermore, APOE is a major protein constituent of several cholesterolenriched lipoproteins, and it is now known that these cholesterol-enriched, APOE-containing lipoproteins are chylomicrons (CMs), VLDL remnants, and a subclass of HDLs. As has been mentioned previously, APOE has extensive roles in the mediation of lipid metabolism including lipoprotein clearance due to its ability to bind to CM remnant receptors. APOE also has a high affinity for the LDL receptor (LDLR; Hayek et al. 1994). In addition, as a component of HDL subclass, APOE participates in the 'reverse cholesterol transport' (RCT) process to remove excess cholesterol from the cells and transport it to the liver for biliary secretion (Trigatti et al. 1999).

Plenty of studies have demonstrated that genetic variation at the Apoe locus is involved in related pathologies of advanced age, is associated with an increased risk of developing atherosclerosis, and determines susceptibility to cardiovascular disease and Alzheimer's disease (Plump \& Breslow 1995, Mahley \& Rall 2000). The deletion of Apoe results in a fatty symptom that is unaffected by age or sex. APOE has multiple functions in steroidogenic cells as well. Dyer \& Curtiss compared the activities of APOE-rich HDLs with those of APOE-poor HDLs and suggested that APOE is responsible for the inhibition of androgen production (Dyer \& Curtiss 1988, Nicosia et al. 1992). Human APOE expressed in the murine $\mathrm{Y} 1$ adrenal cells could inhibit adrenocorticotrophic hormone- and CAMP-stimulated steroidogenesis too (Reyland \& Williams 1991, Reyland et al. 1991). These results suggest that Apoe may be an important modulator of cholesterol utilization in steroidogenic cells. Taking its key role in plasma lipoprotein and cholesterol metabolism into account (Moreno et al. 2006, Ye et al. 2008, Huang et al. 2009), studies have suggested that Apoe might affect steroid production and then affect fertility directly or indirectly. The influence of Apoe deletion on reproduction has not been assessed previously, and it is supposed to have a certain relationship with hormone synthesis and disordered cholesterol metabolism. On the one hand, obesity and abnormal lipoprotein metabolism in Apoe ${ }^{-/-}$mice may affect female fertility and, on the other hand, the Apoe gene itself may have a vital function in the reproductive system.

In this study, we exploited Apoe ${ }^{-/-}$mice to explore the interaction between lipid metabolism and reproduction with an expectation of gaining a better understanding of Apoe and finding a platform for probing into reproduction in human obesity.

\section{Materials and methods}

\section{Animals}

WT C57BL/6J mice were bought from the Chinese Center for Disease Control and Prevention (Beijing, China). Apoe ${ }^{-/-}$ mice of the $\mathrm{C} 57 \mathrm{BL} / 6 \mathrm{~J}$ background were obtained from Jackson Laboratory (B6; 129-Apoe ${ }^{\mathrm{tm} 1 \mathrm{Unc}}$, Bar Harbor, ME, USA). The targeted mutation was created by inserting a neomycin resistance cassette that deleted part of exon 3 and part of intron 3 of the Apoe gene. The mice were manipulated in accordance with the Guidelines of Shandong Normal University for the Care and Use of Laboratory Animals. The mice were provided standard rodent chow and water ad libitum and were maintained under a controlled $12 \mathrm{~h}$ light: $12 \mathrm{~h}$ darkness cycle in a pathogen-free mouse room (Cui et al. 2011).

The genotypes of the mice were determined by PCR analysis, which included the extraction of DNA from mouse tail tissue and agarose gel separation. The sequences $\left(5^{\prime}-3^{\prime}\right)$ of primers used in the identification of Apoe genotype were as follows: P1: GCCTAGCCGAGGGAGAGCCG, P2: TGTGACTTGGGAGCTCTGCAG, and P3: GCCGCCCCGACTGCATCT. The expected product sizes were 245 and 155 bp in the mutant homozygous and WT strains respectively.

For the breeding assay, 8-week-old females were coupled with reproductively proven C57BL/6J males for 6 months (two females per male). Vaginal canals were gently checked for copulatory plugs every morning, and the parturition dates and litter sizes were recorded. Vaginal opening time of the litter was recorded every morning since the day of weaning (D 21). Eightweek-old mice $(n=6)$ were used for estrus cycle detection for 30 days according to vaginal cytology and external appearance (Cui et al. 2011, Tang \& Zhang 2011). Superovulation was induced by s.c. injection of $5 \mathrm{IU}$ pregnant mare serum gonadotropin (PMSG; Ningbo Sansheng Pharmaceutical Co., Ltd., Zhejiang, China) at 24 days of age $(n=12)$. After $48 \mathrm{~h}$, $5 \mathrm{IU}$ human chorionic gonadotropin (hCG) (Ningbo Sansheng Pharmaceutical Co., Ltd.) was injected. The oviducts and ovaries were dissected $14 \mathrm{~h}$ after hCG treatment, and the number of ova in the oviduct was determined. Experimental mice were weighed and killed by cervical dislocation. In the hormone assays, 8-week-old mice $(n=6)$ and D 100 mice $(n=6)$ at diestrus were used for determining estrogen and progesterone concentrations. For the estrogen and progesterone tests, serum was prepared by cardiac puncture with commercially available kits (lodine [125I]-E $E_{2}$ RIA Kit and lodine [ $\left.{ }^{125} \mathrm{I}\right]-$ Prog RIA Kit, Jiuding Biological Engineering Co., Tianjin, China) by RIA. Ovaries were collected in a corresponding manner for different experiments.

\section{Ovarian lipid deposition Oil Red O staining}

Oil Red $\mathrm{O}$ is a dye that detects primarily cholesteryl ester droplets in adrenocortical and ovarian cells (Nicosia et al. 1992, Plump et al. 1996, Trigatti et al. 1999). 
Cryosections (10 $\mu \mathrm{m}$ thick) were prepared from the O.C.T compound (Sakura Finetek, Inc., Torrance, CA, USA)embedded ovary samples collected from mice at D 100 using a freezing microtome (Leica CM-1850, Bensheim, Germany), fixed in $4 \%$ paraformaldehyde (PFA) in PBS for $5 \mathrm{~min}$, and washed and dehydrated with $60 \%$ alcohol for $5 \mathrm{~min}$. Later, the slides were stained using $0.5 \%$ Oil Red O (O0625, SigmaAldrich, Inc.) in $60 \%$ isopropanol for 12 min; after washing in $60 \%$ alcohol for $30 \mathrm{~s}$ two times, the sections were hydrated for $1 \mathrm{~min}$ and then counterstained with hematoxylin. Finally, the sections were mounted with $50 \%$ glycerol. The tissue sections were observed and photographed using an Olympus ML2000 microscope (Olympus).

\section{Transmission electron microscopy (TEM)}

Ovaries collected from 3-month-old mice were cut into $1 \mathrm{~mm}^{3}$ sections on ice and fixed in $3 \%$ glutaraldehyde in $0.2 \mathrm{M} \mathrm{PB}$ (0.874 $\mathrm{g} \mathrm{NaH}_{2} \mathrm{PO}_{4}$ and $5.158 \mathrm{~g} \mathrm{Na}_{2} \mathrm{HPO}_{4}$ in $100 \mathrm{ml} \mathrm{H} \mathrm{H}_{2} \mathrm{O}$ ), and the samples were refixed by osmic acid for a few hours and then processed for transmission electron microscopy (TEM) as described by Yue \& Spradling (1992).

\section{Plasma and ovarian cholesterol and triglyceride analyses}

For the analysis of plasma lipids, at D 18, D 42, and D 100, mice were anesthetized, and blood samples were collected by cardiac puncture $(n=6)$. Lipoproteins in serum were separated using fast-protein liquid chromatography (FPLC; Agilent Technologies, Palo Alto, CA, USA) and fractioned at a constant flow rate of $50 \mu \mathrm{l} / \mathrm{min}$ with PBS. Total plasma cholesterol and triglyceride (TG) concentrations for each individual sample were measured using a colorimetric method with commercially available kits for total cholesterol (TC) and TG concentrations (Sigma Chemical Co.).

For the analysis of ovarian lipids, $80 \mathrm{mg}$ ovarian tissue samples mixed with $1.5 \mathrm{ml}$ chloroform and $0.75 \mathrm{ml}$ methanol were prepared in a centrifuge tube and stirred for $10 \mathrm{~min}$ using a refiner at $2300 \mathrm{~g}$. The supernatants were isolated and then $0.8 \mathrm{ml}$ chloroform and $0.4 \mathrm{ml}$ methanol were added to the precipitates and stirred again. The two supernatants were combined and mixed with $0.54 \mathrm{ml} 145 \mathrm{mM} \mathrm{NaCl}$; after centrifugation at $6000 \mathrm{~g}$ for $5 \mathrm{~min}$, the lower chloroform phase was collected into a weighed EP tube and then discarded. The residuals were weighed for calculation of the total lipid weight in the ovaries. For the TC and TG tests, $10 \mu \mathrm{l}$ chloroform and $990 \mu \mathrm{l} 10 \%$ Triton X-100 (in isopropanol) were added to dissolve lipid and then a colorimetric method with commercial kits mentioned above was used to carry out the tests.

\section{Immunohistochemical analysis of APOE}

Ovaries collected from the WT mice at D 18, D 42, and D 100 were used for the determination of Apoe expression. Cryosections $(10 \mu \mathrm{m}$ thick) were treated in acetone for $10 \mathrm{~min}$ at $-20{ }^{\circ} \mathrm{C}$, followed by rinsing with $0.3 \%$ Triton X-100 in PBS for 30 min. After washing three times with PBS, endogenous peroxidases were quenched with $0.3 \%$ hydrogen peroxide
$\left(\mathrm{H}_{2} \mathrm{O}_{2}\right)$ in methanol for $30 \mathrm{~min}$. The sections were then blocked with $5 \%$ BSA (Sigma-Aldrich) at $37^{\circ} \mathrm{C}$ for $30 \mathrm{~min}$ and incubated overnight at $4{ }^{\circ} \mathrm{C}$ with the primary antibody against APOE (SC-9364, Santa Cruz; 1:200 dilution). After washing with PBS, the sections were treated with a peroxidaseconjugated rabbit anti-goat IgG secondary antibody (SC-2774, Santa Cruz; 1:200 dilution) for $1 \mathrm{~h}$. Positive signals were developed using the DAB kit (ZLI-9031, ZSGB-BIO, Beijing, China), and the sections were counterstained with hematoxylin to visualize nuclei.

\section{Histological evaluations of the ovarian follicles}

Ovaries were collected from the mice at postnatal days 4, 18, 42, and 90 and fixed in 4\% PFA in PBS overnight, and after several washes with PBS, tissue samples were dehydrated and made transparent using Leica-ASP200S Dehydrator (Leica), and then the samples were embedded in paraffin by LeicaEG1150H Embedding Center (Leica). Paraffin serial sections ( $5 \mu \mathrm{m}$ thick) were prepared using Leica-RM2125 (Leica). The sections were then stained with hematoxylin and eosin. The fifth of five sections of the whole serial sections was observed, and the numbers of primordial, primary, preantral, and antral follicles were determined. The classification and computing methods of the follicles have been described earlier (Cui et al. 2011).

\section{RNA extraction and real-time PCR}

Ovaries obtained from neonatal and mature mice at D 4 and D 100 were homogenized in TRIzol Reagent (Tiangen Biotech Co., Ltd, Beijing, China), and then RNA was extracted according to the manufacturer's instructions. Total RNA was treated with RNase-free DNase (Tiangen Biotech Co., Ltd), and then $2 \mu \mathrm{g}$ RNA were used for RT with SuperScript (SS) First-Strand Synthesis System (Tiangen Biotech Co., Ltd).

Real-time PCR was carried out using SYBR Green Master Mix on the Bio-Rad IQ5 Muiltcolor Real-time PCR Detection System (Bio-Rad). The sequences of the primers used are listed in Table 1.

PCR was carried out under the following conditions: 3 min at $95^{\circ} \mathrm{C}$ and 40 cycles of $15 \mathrm{~s}$ at $95^{\circ} \mathrm{C}, 30 \mathrm{~s}$ at $60^{\circ} \mathrm{C}$, and $30 \mathrm{~s}$ at $72{ }^{\circ} \mathrm{C}$. Melting-curve analyses were carried out to verify product identity. The PCR products were processed in triplicate, and values obtained are expressed relative to the abundance of endogenous $\beta$-actin in the same sample. The amplification efficiency of PCR was analyzed using the LinRegPCR 11.0 software (Academic Medical Center, Amsterdam, The Netherlands), and data were computed based on a calibrator sample using the $\Delta \Delta C$ t method (Pfaffl 2001).

\section{Determination of apoptotic cells by TUNEL assays}

To determine the development of follicles, TUNEL assays were carried out using 42-day-old mice by means of a commercial kit (In Situ Cell Detection Kit, POD, Roche) according to the manufacturer's instructions. Briefly, cryosections (10 $\mu \mathrm{m}$ thick) were fixed in 4\% PFA in PBS for $30 \mathrm{~min}$, and then the slides were rinsed with PBS blocking solution $\left(3 \% \mathrm{H}_{2} \mathrm{O}_{2}\right.$ in methanol) 
Table 1 Target transcripts and primer sequences used for the quantification of mRNA levels in the ovaries.

\begin{tabular}{lllc}
\hline Transcripts & Forward $\left(5^{\prime} \rightarrow 3^{\prime}\right)$ & Reverse $\left(5^{\prime} \rightarrow 3^{\prime}\right)$ & Amplicon size $(\mathrm{bp})$ \\
\hline Actb & GGCTGTATTCCCCTCCATCG & CCAGTTGGTAACAATGCCATGT & 154 \\
A $k$ t 1 & CCTTTATTGGCTACAAGGAACGG & GAAGGTGCGCTCAATGACTG & 164 \\
Bad & AGACGCTAGTGCTACAGATAG & CTCCTCCTCCATCCCTTCAT & 214 \\
Cyp $19 \mathrm{a} 1$ & TCGTCGCAGAGTATCCAGAGG & CGCATGACCAAGTCCACAACA & 163 \\
Hsd $3 b$ & GGTTTTGGGGCAGAGGATCA & GGTACTGGGTGTCAAGAATGTCT & 168 \\
Mtor & TTGGACGGTGTAGAACTTGGA & GTGAGATGTTGCCTGCTTGAT & 234 \\
$N$ f $k b$ & AAGCAGGAAGATGTAGTAGAG & CTATGTGGATGGCATTTAGAC & 196 \\
$P t e n$ & TGGATTCGACTTAGACTTGACCT & GCGGTGTCATAATGTCTCTCAG & 180 \\
$T s c 2$ & CTTGACCAGATTCCATCCTAT & CATACATCCAGTCCACCTAAG & 200 \\
\hline
\end{tabular}

after washing with PBS and incubated with a permeabilization solution $(0.1 \%$ Triton $\mathrm{X}-100$ in $0.1 \%$ sodium citrate) for $2 \mathrm{~min}$ on ice. After several washes with PBS, the sections were incubated with the TUNEL reaction mixture in a humid chamber for $60 \mathrm{~min}$ at $37^{\circ} \mathrm{C}$, and then the sections were incubated with Converter-POD for $30 \mathrm{~min}$ at $37^{\circ} \mathrm{C}$. Positive signals were developed with DAB (ZLI-9031, ZSGB-BIO), and the sections were then counterstained with hematoxylin to visualize nuclei.

\section{Statistical analyses}

Data were analyzed using SPSS statistical software (SPSS, Inc.). Differences among groups were analyzed using one-way ANOVA; values were considered to be significantly different if $P \leq 0.05$. All numerical data are presented as means \pm s.E.M., and all experiments were repeated at least three times.

\section{Results}

\section{Genotype identification of $\mathrm{Apoe}^{-/-}$mice}

The Apoe ${ }^{-/-}$mice were of a homozygous strain. As shown in Fig. 1, the WT mice yielded one fragment of $155 \mathrm{bp}$ (lane 2) and the Apoe mutant mice a fragment of $245 \mathrm{bp}$ (lane 3).

\section{Effect of Apoe deletion on weight}

Since the deletion of Apoe influences cholesterol metabolism and induces obesity apparently, the body weight of the WT and Apoe ${ }^{-/-}$mice as well as their ovary weight was measured. The results showed that Apoe knockout mice presented an increasing tendency towards weight gain and were heavier compared to their counterparts no matter the body weight (Fig. 2A) or the ovary weight (Fig. 2B) during the testing period.

\section{Ovarian fat deposit Oil Red $O$ staining and TEM}

To determine whether the overweight in ovaries was caused by lipid accumulation, cryosections were stained with Oil Red O. The ovaries of WT mice (Fig. 3A) exhibited prominent staining in interstitial cells and theca cells, but staining was much less abundant in granulosa cells. By contrast, greatly increased staining of interstitial cells was observed in the ovaries of Apoe $^{-1-}$ mice (Fig. 3B), reflecting an abundance of lipid storage. A transmission electron microscope was used to observe differences in the microstructures of the two types of mice. The examination confirmed an abundance of lipid droplets in the ovarian cells of $A p o e^{-/-}$mice (Fig. 3D); furthermore, their endoplasmic reticulum (ER) had become swollen and tubby (Fig. 3F) compared with that of their counterparts (Fig. $3 \mathrm{E}$ ), indicating that there may be a dysfunction of the organelles.

\section{Plasma and ovarian cholesterol and TG analyses}

To investigate the influence of Apoe knockout on lipid metabolism, the different classes of plasma lipoproteins were separated and the changes were analyzed by FPLC analysis. When the lipoproteins were analyzed using TC kits (Fig. 4A, C, and E), HDLs (peaking at around fraction number 35) were found to be the major cholesterolcontaining particles in the WT mice and were present at lower levels in fractions 1-30, which normally included VLDLs and LDLs. By contrast, there was a significant increase in TC content in VLDL and LDL particles in the

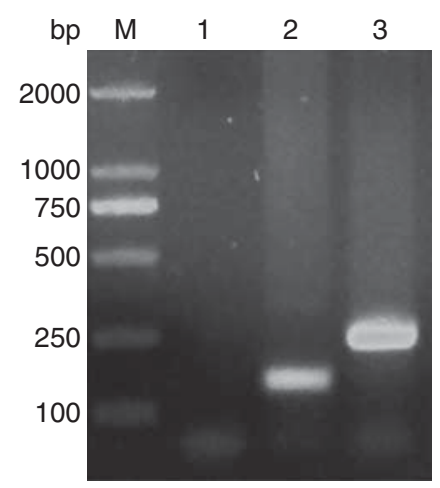

Figure 1 Genotype identification of the Apoe ${ }^{-/-}$mice. Genomic DNA samples from the Apoe-knockout and WT mice were amplified by standard PCR, and the genotype was identified by the size of the amplicon (WT mouse: 155 bp and mutant strain: 245 bp). M: DL2000 marker; lane 1: negative control; lane 2: WT mouse; lane 3: Apoe $^{-/-}$mouse. 

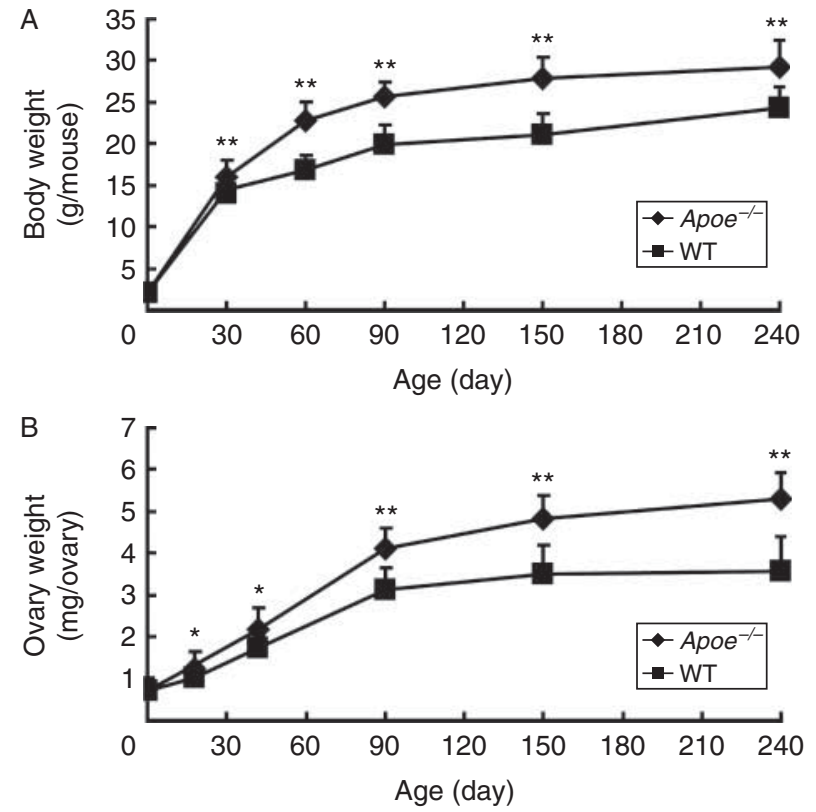

Figure 2 Comparison of the weights of the WT (square) and Apoe (diamond) female mice. (A) Body weight. (B) Ovary weight. Bars represent means \pm S.E.M. $\left(n=11\right.$ mice per genotype). ${ }^{*} P<0.05$, $* * P<0.01$.

Apoe $^{-/-}$mice, but that in HDL particles (fractions 31-45) decreased after D 18 inversely. Similarly, when lipoproteins were subjected to TG assays (Fig. 4B, D, and $\mathrm{F}$ ), the number of VLDL fractions was also found to be markedly increased in the Apoe ${ }^{-/-}$mice after birth, but there was little change in that of the LDL and HDL fractions. Although TG concentrations decreased with age, they were always higher than those of the WT mice (Fig. 4B, D, and F).

To detect the effects of plasma lipoprotein variation on inner ovarian lipids, we measured ovarian TC and TG concentrations. The results demonstrated that although the deletion of Apoe markedly increased blood TC and TG concentrations, it had no significant influence on ovarian TG or TC concentrations (Fig. 4G and H).

\section{Expression of Apo in the ovaries}

We examined the immunostaining of APOE protein in ovarian follicles obtained at D 18, D 42, and D 100. The results indicated a high expression in interstitial cells, theca cells, and oocytes of primary and secondary follicles (Fig. 5A) and antral follicles (Fig. 5B), but not in granulosa cells. Furthermore, there was a marked expression of APOE in CL (Fig. 5C). There was no difference in the location of the expression of APOE protein among the mice at different ages (the lower right corner of Fig. 5A, B, and C). There was no detectable immunostaining in the ovaries of $A p o e^{-/-}$mice as predicted (Fig. 5D).

\section{Influence of Apoe deletion on ovarian follicle development}

Since APOE protein was highly expressed in ovarian follicles, ovarian histomorphology was examined to determine whether APOE played a role in ovarian follicle development. Compared with the WT mice, the Apoe ${ }^{-/-}$mice had more follicles at birth (Fig. 6A), but with increasing age the number of total follicles decreased rapidly. At D 42, the two groups had an equal number of follicles, but later, the number of total follicles was much lower in the Apoe ${ }^{-1-}$ mice (Fig. 6A).

At D 4, there were three types of follicles in the WT mice: primordial follicles, some primary follicles, and a few preantral follicles, the numbers of which were significantly lower than those in the $A p o e^{-/-}$mice (Fig. 6B, C, and D). Antral follicles were detected at D 18 (Fig. 6E). Although there was no considerable difference
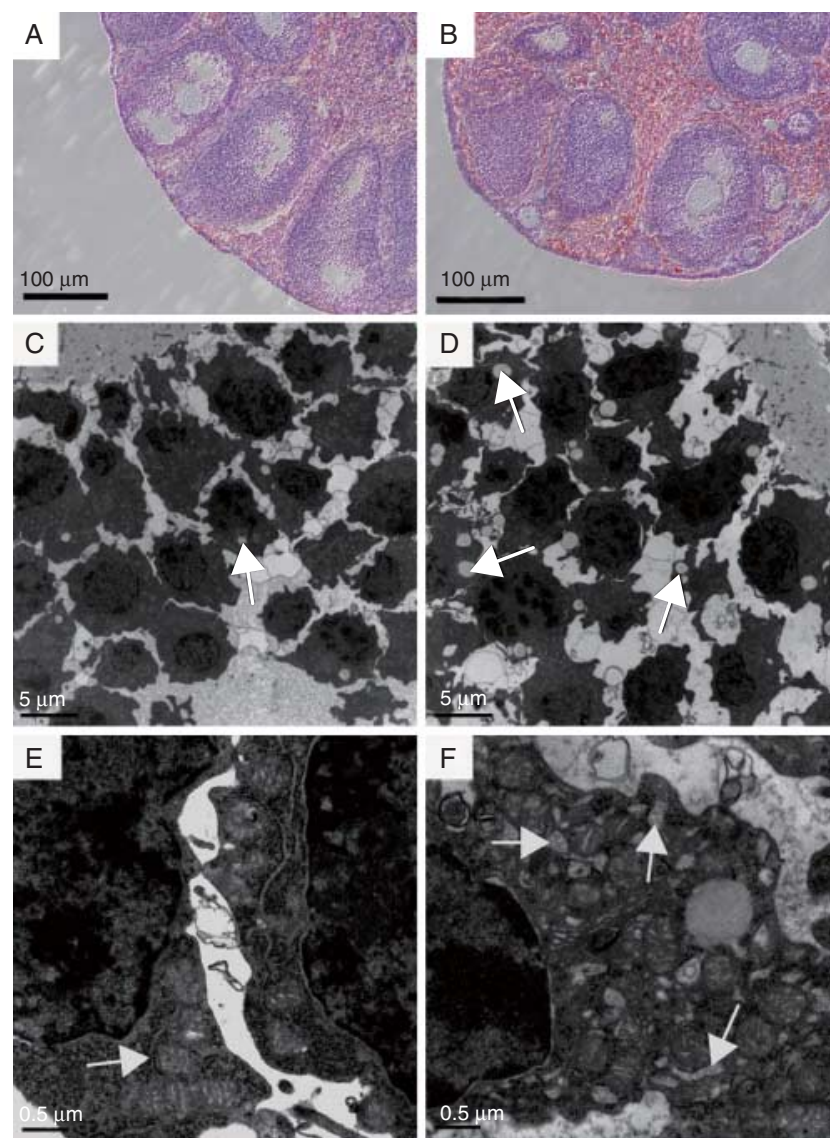

Figure 3 Ovarian fat deposit and transmission electron microscope detection. Typical Oil Red O staining of lipids in ovaries of D 100 WT female mice (A) and Apoe ${ }^{-/-}$mice (B). (C, D, E, and F) Electron micrographs of ovaries showing an increased number of lipid droplets (black arrows) in the Apoe ${ }^{-/-}$mice (D) compared with the WT mice (C). (E and F) Enlargements of the electron micrographs (C and D), showing clusters of organelles and abnormally enlarged endoplasmic reticulum (white arrows) in the Apoe ${ }^{-/-}$mice (F) compared with the age-matched WT mice (E). 

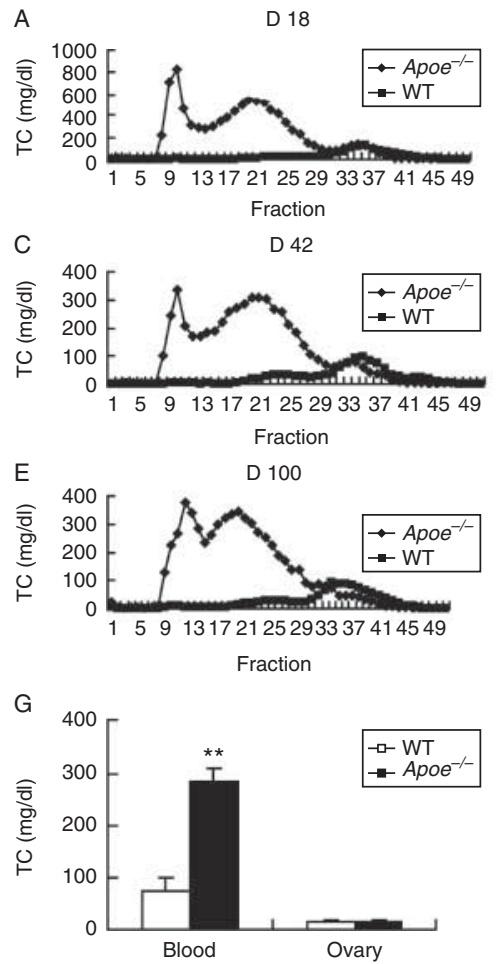

B

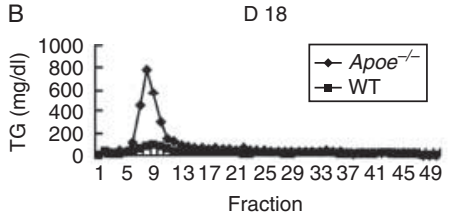

D

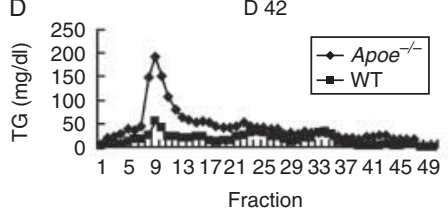

$\mathrm{F}$

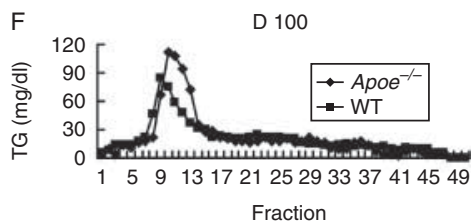

$\mathrm{H}$

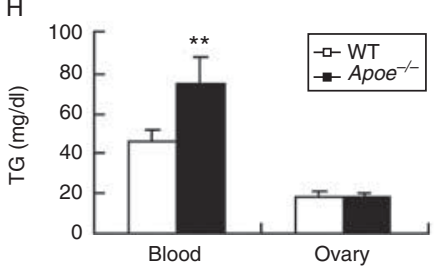

Figure 4 Plasma and ovarian TC and TG analyses of the WT and Apoe ${ }^{-/-}$mice. (A, B, C, D, E, and F) FPLC profiles of TC and TG. Plasma pooled from six female mice of each genotype was subjected to FPLC analysis. TC and TG concentrations in each fraction were measured. (A) TC concentration in mice at D 18. (B) TG concentration in mice at D 18. (C) TC concentration in mice at D 42. (D) TG concentration in mice at D 42. (E) TC concentration in mice at $\mathrm{D}$ 100. (F) TG concentration in mice at D 100. (G and H) TC and TG concentrations in plasma (left histogram) and ovarian homogenate (right histogram) in the WT (white bar) and Apoe ${ }^{-1-}$ (black bar) mice. Bars represent mean \pm s.E.M. $(n=6)$. **Indicates significant differences at $P<0.01$. in the number of primordial follicles and primary follicles in the Apoe ${ }^{-1-}$ mice compared with that in the WT mice at D 42 (Fig. 6B and C), more follicles were detected at other time periods. However, at D 100, not only the number of total follicles but also that of the four types of the follicles decreased due to the deletion of Apoe (Fig. 6A, B, C, D, and E).

\section{Expression of Cyp19a1, Hsd3b, and phosphoinositide 3-kinase pathway-related genes}

Since the number of total follicles in the $A p o e^{-/-}$mice at D 4 and D 18 exceeded that in the WT strains in contrast to the D 100 profile, Cyp19a1, Hsd3b, and a series of phosphoinositide 3-kinase (PI3K) pathway genes were investigated, including Akt1, Bad, Mtor, $N f k b$, Pten, and Tsc2, by real-time PCR. The results demonstrated that the expression of $\mathrm{Hsd} 3 \mathrm{~b}$ and Mtor increased significantly at D 4 in the mutant strains (Fig. 7A). At D 100, while the expression of Cyp19a1, $\mathrm{Hsd} 3 \mathrm{~b}$, Mtor, and Nfkb decreased significantly, that of $\mathrm{Bad}$ and TsC2 increased dramatically in the mutant mice (Fig. 7B).

\section{TUNEL}

In order to validate the statistics of ovarian follicles, TUNEL experiment was used to ascertain the direction in which the lost follicles had gone (Fig. 8). There were more TUNEL-positive follicles (Fig. 8C) in the ovaries of
Apoe ${ }^{-/-}$mice (Fig. 8B) than in those of the WT mice (Fig. 8A). The result indicated a higher apoptosis index in the Apoe $^{-/-}$mice $(21.2 \%)$ than in the WT mice $(17.5 \%)$, suggesting the presence of high-speed atretic follicles (Fig. 8E).

\section{Fertility assays}

We examined various physiological features of reproduction to determine whether there are some differences between the WT and Apoe ${ }^{-/-}$mice. The results indicated that serum estrogen (Fig. 9A) and progesterone (Fig. 9B) concentrations were significantly decreased in the plasma of Apoe ${ }^{-/-}$mice at D 100, although similar to those of the WT mice at D 56. However, the number of ova shed by the Apoe $^{-/-}$mice was almost equal to that shed by the WT mice (Fig. 9C), and the deletion of Apoe did not affect the vaginal opening time (Fig. 9D). The Apoe ${ }^{-/-}$female mice displayed decreased frequency and duration of estrus (Fig. 9E). As for the fertility during a 6-month breeding period, the total number of pups, the litters, and the average pups per litter were not significantly different between WT and Apoe ${ }^{-1-}$ females (Fig. 9F).

\section{Discussion}

The present study demonstrates that obesity caused by Apoe gene deletion has no significant influence on the fertility of mice. The number of pups born to the Apoe $^{-1-}$ mice was similar to that born to the WT mice in 


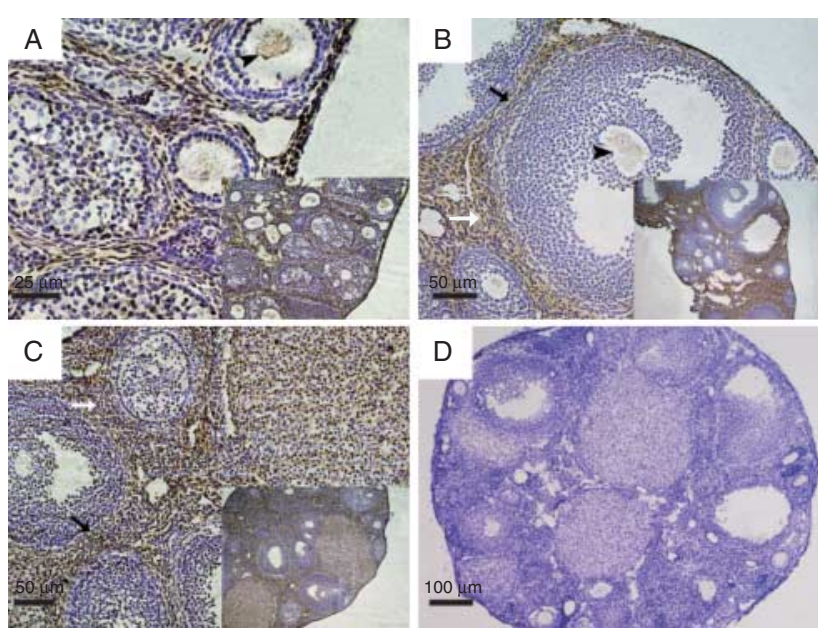

Figure 5 Immunohistochemical localization of APOE protein during ovarian follicle development. (A, B, and C) Cryosections of the ovaries of WT mice at D 18, D 42, and D 100 respectively. Positive staining is shown as brown color in the ovarian sections. (D) Ovarian section of an Apoe $^{-1-}$ mouse exhibiting no positive staining. Black arrows, interstitial cells; white arrows, theca cells; and arrowheads, oocytes.

6 months. Although plasma cholesterol metabolism was disordered, lipids accumulated, estrus frequency and duration decreased, and estrogen as well as progesterone concentrations declined at D 100 in the Apoe ${ }^{-/-}$mice compared with their WT counterparts; there was no significant difference in their ovulation and fertility rates.

Our findings are in conflict with the traditional opinion that obesity and overweight affect fertility and may even cause infertility due to anovulation and menstrual irregularities (Trigatti et al. 1999, Brannian 2011). The reason for this might be that obesity caused by Apoe deletion is not serious enough and the increase in weight is not too severe compared with the standard criteria. We observed that Apoe knockout led to an increase in body weight as well as ovary weight. Oil Red $\mathrm{O}$ staining revealed the existence of excess lipids, and TEM indicated the presence of more lipid droplets around the oocytes, which were considered to be transformed by the redundant lipids. Furthermore, FPLC analysis of the lipoproteins indicated that the increased total plasma cholesterol content in the Apoeknockout mice was a consequence of a dramatic increase in the cholesterol content in VLDL and VLDLsized LDL particles, and our results are consistent with those of the former studies (Plump et al. 1992, Zhang et al. 1992). Further studies carried out by these authors have demonstrated that the VLDL fractions had a much greater ratio of $\mathrm{APOB} 48$ to $\mathrm{APOB} 100$ than those in normal animals. APOB48 is a cell-surface protein of CMs and APOB100 functions as an Apo in VLDLs and LDLs. Therefore, these results suggest that the particles accumulated in the very-low-density fraction of the mutants were not normal VLDLs, but probably the remnants of CMs or VLDLs, as the lack of Apoe made them lose the ability to bind to the CM receptor to be cleared. Moreover, compared with those in the WT mice, VLDL fractions in the Apoe ${ }^{-/-}$mice were found to contain substantial amounts of Apoa 1 and Apoa4, both of which were low in these fractions in the WT mice (Zhang et al. 1992). We also found that the cholesterol content of HDLs was decreased. The reduced HDL concentrations may also reflect the absence of APOE-containing HDL particles. The reason for the decreased HDL concentrations is neither reduced biosynthesis nor accelerated catabolism of APOA1, but is the redistribution to remnant particles as a structural component in the absence of Apoe (France et al. 1989, Zhang et al. 1992,
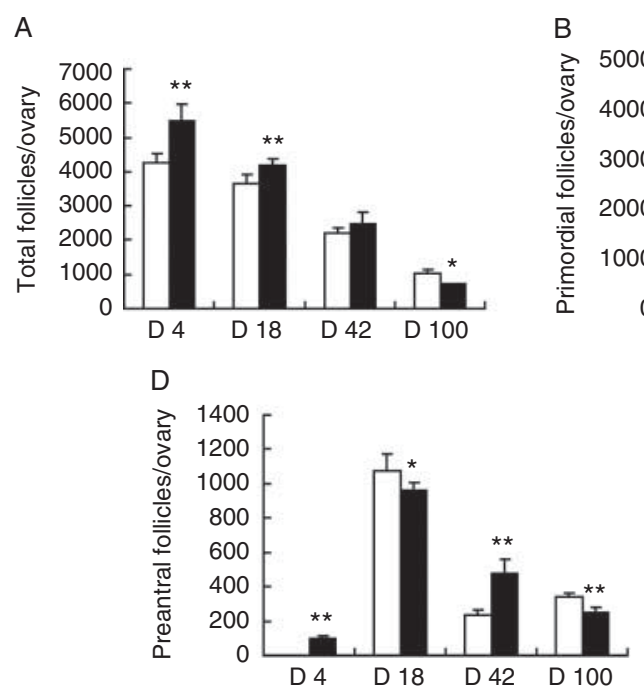
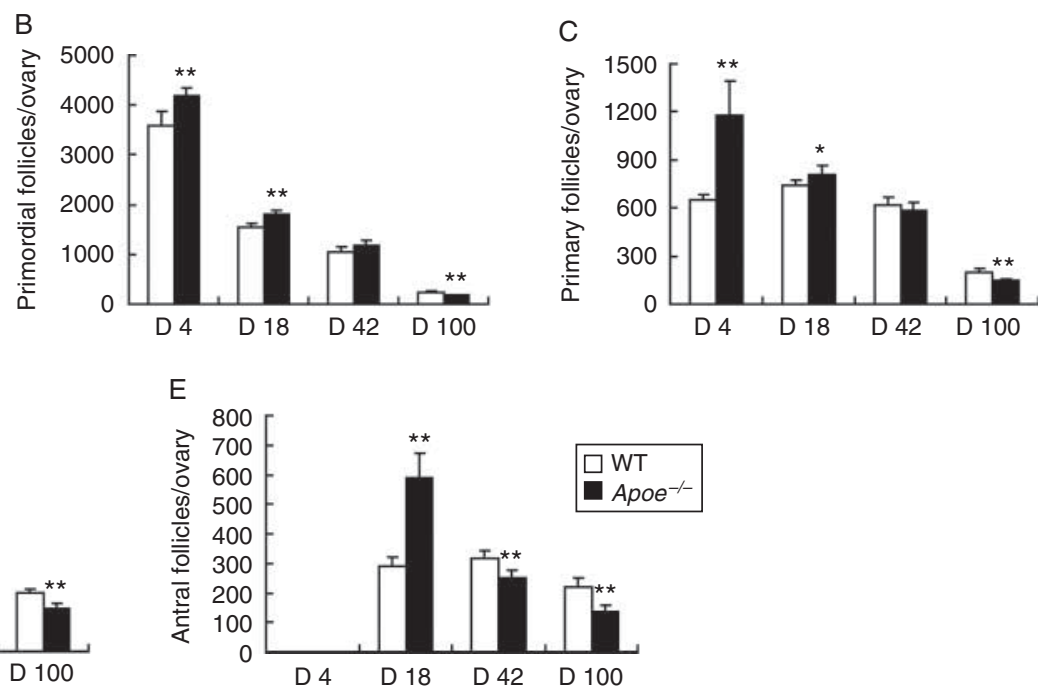

Figure 6 Effect of Apoe deletion on the number of ovarian follicles at different ages. (A) Number of total follicles per ovary. (B) Number of primordial follicles per ovary. (C) Number of primary follicles per ovary. (D) Number of preantral follicles per ovary. (E) Number of antral follicles per ovary. WT mice (white bars) and Apoe ${ }^{-1-}$ mice (black bars). Bars represent mean \pm s.E.M. $(n=6) .{ }^{*} P<0.05, * * P<0.01$. 

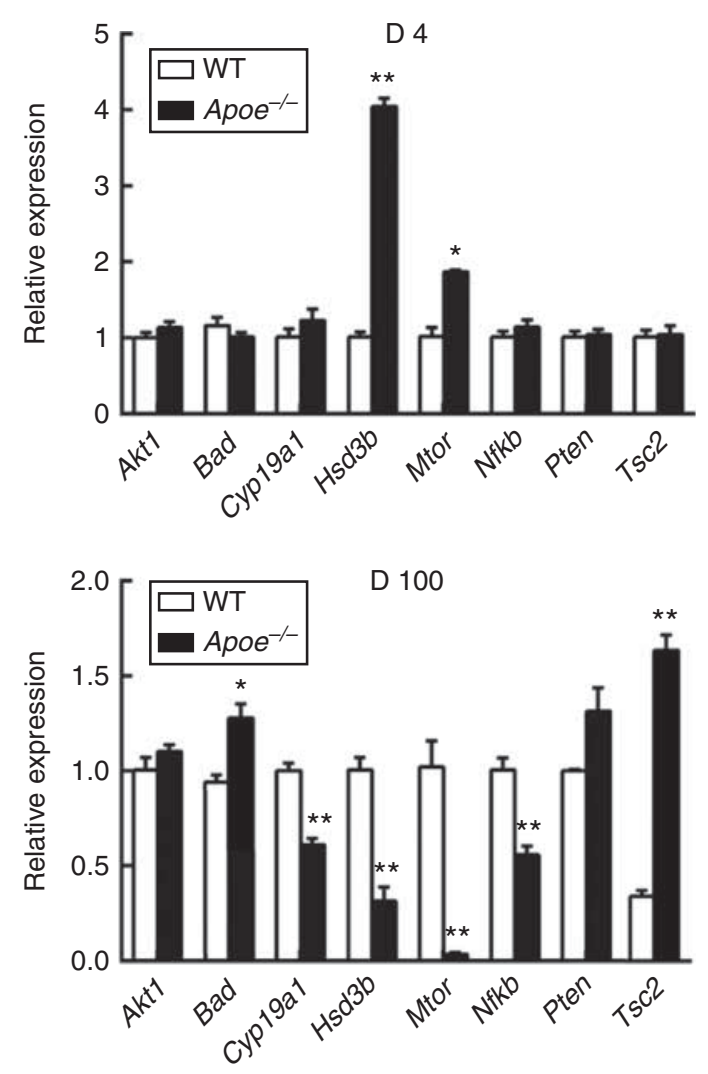

Figure 7 Real-time PCR analysis of Hsd3b, Cyp19a1, and PI3K pathway-related genes, Akt1, Bad, Mtor, Nfkb, Pten, and Tsc2. Three independent assays were carried out for each gene. The data represent means \pm S.E.M. of triplicate simples. ${ }^{*} P<0.05 ;{ }^{* *} P<0.01$.

Trigatti et al. 1999). Low HDL concentrations have been found to be associated with decreased unidirectional transportation of cholesterols from peripheral tissues, and the redundant cholesterols cannot be metabolized by the liver for excretion (Glomset 1968).

Tissues synthesize cholesterol and uptake LDLs/HDLs from the plasma for their steroidogenesis and at the same time clear redundant cholesterols in the pattern of HDLs reversely (Goldstein \& Brown 1985, Connelly \& Williams 2004). APOE functions as a subclass composition of HDLs and is responsible for binding to the cell-surface receptor Scarb B1 (SR-B1) directly in the selective uptake of HDL cholesteryl esters (Arai et al. 1999); the loss of Apoe blocks the efflux of APOE-HDL cholesterol from the ovaries and induces lipid accumulation. Therefore, the reason for the increased weight of Apoe $^{-/-}$ovaries might be also due to the inefficient RCT process. A study carried out in Apoe ${ }^{-/-}$mice has shown a marked increase in APOB100 content in serum, consistent with the increased serum LDL cholesterol content and Apob100 mRNA content in the liver (Ye et al. 2008). The data indicate that the deletion of Apoe might activate the LDL uptake pathway as a remediation for tissue cholesterol usage and blood lipid clearance. Therefore, the compensated uptake pathway and diminished RCT pathway might exaggerate lipid accumulation in the ovaries.

Mouse APOE is localized in the theca, interstitial cells, and oocytes as well as in $\mathrm{CL}$, as indicated in the previous reports (Nicosia et al. 1992, Bogan \& Hennebold 2010). The APOE protein synthesized in theca cells, interstitial cells, and $\mathrm{CL}$ cells may act in an autocrine fashion to influence androgen and progesterone synthesis. The diverse sites of Apoe expression may indicate multiple roles in ovarian metabolism. There were no obvious gross morphological abnormalities in the ovaries of Apoe-knockout mice. Histochemical analysis of the ovaries demonstrated that there were more follicles in the Apoe $e^{-/-}$mice at birth and an increased number of preantral follicles at D 4 and of antral follicles at D 18 and that at D 100 the total number of follicles reduced sharply. These results indicate that the deletion of Apoe has an obvious influence on ovarian follicle development. Previous studies have demonstrated that intraovarian APOE can control the production of
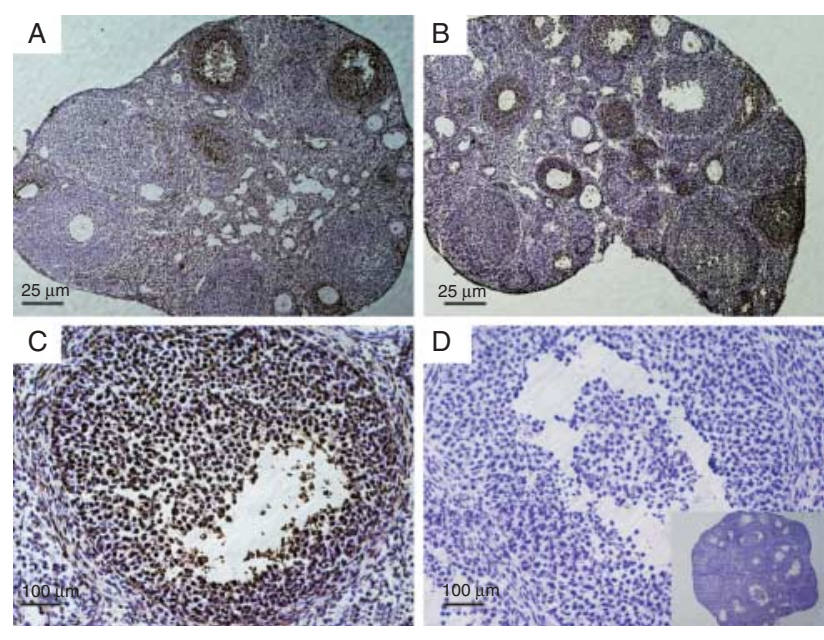

E

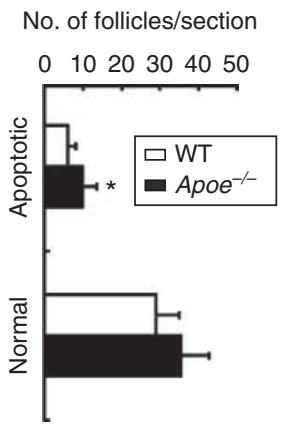

Figure 8 Representative photographs of TUNEL-stained (brown) ovarian sections. Forty-two-day-old mice were used. (A) WT ovary. (B) Apoe ${ }^{-1-}$ ovary. (C) A characteristic atretic follicle. (D) Negative control.

(E) Ovarian sections of the Apoe ${ }^{-/-}$mice showing more apoptotic follicles compared with those of the WT mice. Six sections were prepared from three WT mice and eight sections from five Apoe ${ }^{-1-}$ mice. Bar represents mean \pm S.E.M. ${ }^{*} P<0.05$. 

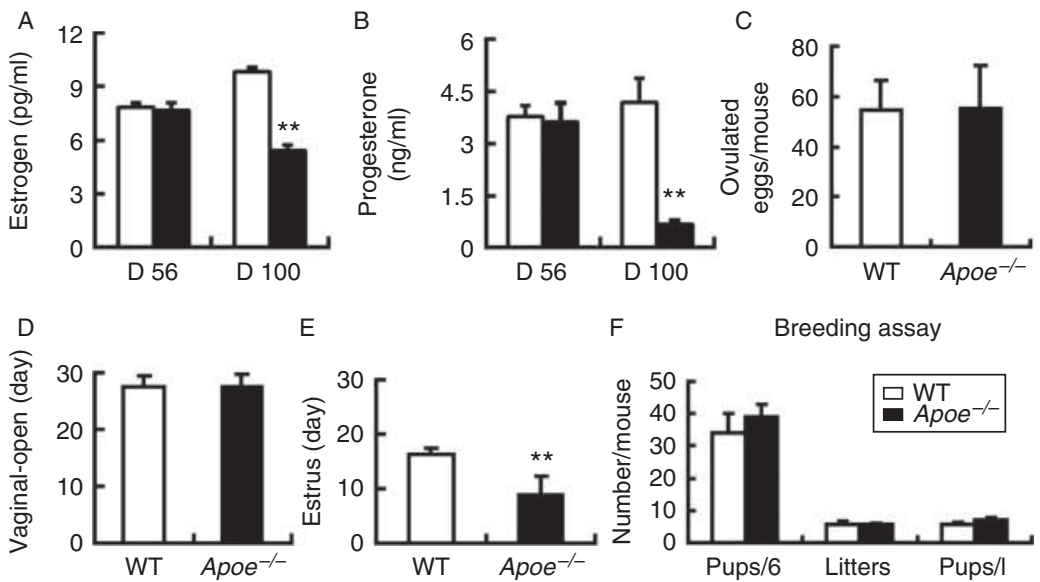

E

F

Breeding assay

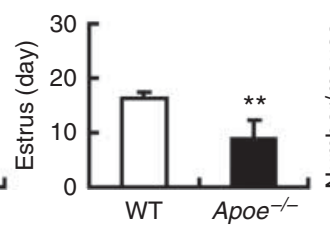

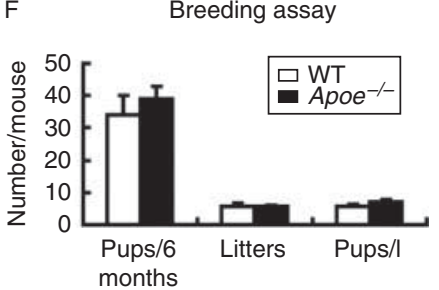

Figure 9 Physiological characteristics of reproduction of the WT (white bars) and Apoe ${ }^{-/-}$mice (black bars). (A and B) Serum estrogen and progesterone concentrations of mice at diestrus stage at D 56 and D 100 respectively $(n=6)$. (C) Ova recovered at $14 \mathrm{~h}$ after the ovulatory stimulus from oviducts of mice at D 24 $(n=10)$. (D) Vaginal opening time detected from the time of weaning $(n=8)$. (E) Mean length of the occurrence of cell populations in vaginal smears indicative of estrus in WT and Apoe ${ }^{-/-}$mice at D 56 $(n=6)$. (F) Number of pups and litters born per female and the average number of pups per litter, which did not differ during the 6-month breeding period from D $56(n=6)$. Bar represents mean \pm S.E.M. ${ }^{* *}$ Indicates significant differences at $P<0.01$. androgen in theca cells (Zerbinatti et al. 2001). Therefore, the level of androgens in the Apoe ${ }^{-/-}$mice may exceed that in the WT mice. HSD3B is involved in the biosynthesis of androgens in ovaries (Lavoie \& King 2009). Our qPCR analysis also showed that the expression of $\mathrm{Hsd} 3 \mathrm{~b}$ increased significantly in the Apoe $^{-/-}$mice at D 4. Androgens can increase the level of FSHR mRNA at all stages of primate ovarian follicle development (Weil et al. 1999), and the FSH pathway can promote the growth of follicles by driving the proliferation and differentiation of granulosa cells (Sasson et al. 2003). Testosterone treatment of immature hypophysectomized rats has been shown to lead to an increase in apoptotic DNA fragmentation in granulosa cells of early antral and preantral follicles rather than in those of primordial and primary follicles (Billig et al. 1993). So in Apoe ${ }^{-/-}$mouse ovaries, the excess androgen may play a positive role in early follicular development, which may be an important reason for the total number of follicles to increase significantly at D4 compared with that of WT animals. The qPCR analysis also revealed that the expression of Cyp19a1 and $\mathrm{Hsd} 3 \mathrm{~b}$ in the Apoe ${ }^{-/-}$mice decreased significantly at D 100. HSD3B and CYP19A1 are essential for the biosynthesis of progesterone and estrogen (Mendelson et al. 1990, Lavoie \& King 2009), so the production of hormones is depressed in response to lower enzyme concentrations, and this is consistent with our hormone detection results indicating that serum estrogen and progesterone concentrations were inferior to those of the WT mice at D 100. Higher androgen and lower estrogen concentrations may cause follicle atresia. In addition, recent evidence suggests that obese animals exhibit increased ER stress in the liver and adipose tissue (Jo et al. 2012). ER can sense and transduce apoptotic signals. Various genetic and environmental stresses interfering with protein folding in ER can induce ER stress to regulate cell differentiation to survive nutrient-limiting conditions or to produce large amounts of secreted products such as hormones, antibodies, or growth factors (Kaufman et al. 2002, Oyadomari et al. 2002). ER stress is closely associated with lipid homeostasis, and intracellular accumulation of cholesterol in the ER can reversibly inhibit protein transport and secretion (Kockx et al. 2012). Long-term ER stress will lead to cell apoptosis, and ER swell is the major step in apoptosis. The abnormal ER appearing in Apoe ${ }^{-/-}$ovarian cells may lead damaged ovarian follicles to atresia.

The PI3K signaling pathway participates in the regulation of the cell cycle, cell metabolism, and cell survival in a variety of tissues (Cantley 2002). Phosphatase and tensin (PTEN), a lipid phosphatase, reverses this process, and so PTEN is a negative regulator in the PI3K pathway (Maehama \& Dixon 1999, Reddy et al. 2008). AKT1, one kind of mammalian isoform of AKT (Coffer et al. 1998), is a target of PI3K and plays a role in its phosphorylated form (Margaret \& Dario 2001, Cantley 2002). MTOR and NFKB, which contribute to cell growth, are regulated by AKT (Madrid et al. 2001, Fingar \& Blenis 2004), while BAD and TSC2 are negative regulators of cell growth and are also regulated by AKT1 (Fingar \& Blenis 2004, Wang et al. 2007). Our qPCR analysis demonstrated that the expression of Mtor and $N f k b$ decreased significantly and that although the expression of $A k t 1$ and Pten did not increase significantly in the mutant mice at D 100, the lower expression may cause decreased follicle activation. It also demonstrated that the notably increased expression of Bad and TsC2 in the $A$ poe ${ }^{-/-}$mice might induce more atresia, which is consistent with our TUNEL results.

Although serum TC and TG concentrations in the Apoe ${ }^{-/-}$mice were higher than those in their counterparts and lipids were accumulated in the ovaries, TC and TG concentrations in the ovaries did not differ significantly. Previous studies have explored the effects of lecithin cholesterol acyltransferase (LCAT), acyl coenzyme A cholesterol acyltransferase (ACAT), and APOA on adrenals (Meiner et al. 1996, 
Plump et al. 1996, Ng et al. 1997). These studies showed that the deletion of Lcat, Acact, and Apoa, which are all important to cholesterol metabolism, affects lipid storage severely, but results in a normal corticosteroid response. Why was there no change in ovarian TC and TG concentrations, despite the presence of more lipid in the ovaries of $A p o e^{-1-}$ mice? In fact, although APOE is the most important supplier of the cholesterol precursor for steroid hormone production in steroidogenic tissues, it is unrelated to extracellular lipid transport (Mahley \& Rall 2000, Zofkova et al. 2002). The lipid droplets appear to be complex, metabolically active organelles that are directly involved in membrane traffic and possibly phospholipid recycling. Although the major components of lipid droplets are cholesterol, cholesterol esters, and TGs in animal cells, using a combination of mass spectrometry and immunoblotting, the Southwestern Medical Center University of Texas identified nearly 40 specifically associated proteins in droplets isolated from Chinese hamster ovarian K2 cells growing in a normal medium (Liu et al. 2004). The proteins can be categorized into five groups according to their functions based on the protein profile. So, the roles of the induced lipid droplets seem to be subtle, but they should be taken into account.

Overweight and obesity are significant and increasing health problems associated with increased risks of morbidity and metabolic and reproductive health consequences. In women, being overweight or obese is associated with impaired fertility and decreased chances of conception in both natural and assisted reproductive technology births. The hypothesis that Apoe may play a role in reproductive functions was confirmed by studies reporting an effect of Apoe common polymorphism on serum concentrations of testosterone and DHEA in postmenopausal women (Zofkova et al. 2002) and by other studies describing a differential fertility associated with common Apoe genotypes in two European populations (Gerdes et al. 1996). However, in this study, obesity induced by Apoe knockout did not have a significant effect on the fertility of female mice. That disordered lipid metabolism had no significant influence on ovarian function might suggest that the overweight has a functional threshold and that, within the threshold, the excess lipid causes little harm to fertility.

\section{Declaration of interest}

The authors declare that there is no conflict of interest that could be perceived as prejudicing the impartiality of the research reported.

\section{Funding}

This work was supported by the NSFC (31172040) and NSF of Shandong (ZR2011CM047), and SRF for ROCS, SEM, as well as $12 \mathrm{DZ} 2260600$ to $\mathrm{C}$ Zhang.

\section{References}

Arai T, Rinninger F, Varban L, Fairchild-Huntress V, Liang CP, Chen W, Seo T, Deckelbaum R, Huszar D \& Tall AR 1999 Decreased selective uptake of high density lipoprotein cholesteryl esters in apolipoprotein E knock-out mice. PNAS 96 12050-12055. (doi:10.1073/pnas.96.21. 12050)

Bellver J, Melo MA, Bosch E, Serra V, Remohi J \& Pellicer A 2007 Obesity and poor reproductive outcome: the potential role of the endometrium. Fertility and Sterility 88 446-451. (doi:10.1016/j.fertnstert.2006.11.162)

Billig H, Furuta I \& Hsueh AJ 1993 Estrogens inhibit and androgens enhance ovarian granulosa cell apoptosis. Endocrinology 133 2204-2212. (doi:10.1210/en.133.5.2204)

Bogan RL \& Hennebold JD 2010 The reverse cholesterol transport system as a potential mediator of luteolysis in the primate corpus luteum. Reproduction 139 163-176. (doi:10.1530/REP-09-0005)

Brannian JD 2011 Obesity and fertility. South Dakota Medicine 64 251-254.

Cantley LC 2002 The phosphoinositide 3-kinase pathway. Science 296 1655-1657. (doi:10.1126/science.296.5573.1655)

Coffer PJ, Jin J \& Woodgett JR 1998 Protein kinase B (c-Akt): a multifunctional mediator of phosphatidylinositol 3-kinase activation. Biochemical Journal 335 1-13.

Connelly MA \& Williams DL 2004 Scavenger receptor BI: a scavenger receptor with a mission to transport high density lipoprotein lipids. Current Opinion in Lipidology 15 287-295. (doi:10.1097/00041433200406000-00008)

Cui LL, Yang G, Pan J \& Zhang C 2011 Tumor necrosis factor $\alpha$ knockout increases fertility of mice. Theriogenology 75 867-876. (doi:10.1016/j. theriogenology.2010.10.029)

Dasgupta S, Sirisha PV, Neelaveni K, Anuradha K, Sudhakar G \& Reddy BM 2012 Role of luteinizing hormone $\beta$-subunit gene variants among South Indian women with polycystic ovary syndrome. Gene 494 51-56. (doi:10.1016/j.gene.2011.11.054)

Devlieger R, Guelinckx I \& Vansant G 2008 The impact of obesity on female reproductive function. Obesity Reviews 9 181-182 author reply 183. (doi:10.1111/j.1467-789X.2007.00460.x)

Dyer CA \& Curtiss LK 1988 Apoprotein E-rich high density lipoproteins inhibit ovarian androgen synthesis. Journal of Biological Chemistry $\mathbf{2 6 3}$ 10965-10973.

Fingar DC \& Blenis J 2004 Target of rapamycin (TOR): an integrator of nutrient and growth factor signals and coordinator of cell growth and cell cycle progression. Oncogene 23 3151-3171. (doi:10.1038/sj.onc. 1207542)

France DS, Hughes TE, Miserendino R, Spirito JA, Babiak J, Eskesen JB, Tapparelli C \& Paterniti JR Jr 1989 Nonimmunochemical quantitation of mammalian apolipoprotein A-I in whole serum or plasma by nonreducing gel electrophoresis. Journal of Lipid Research 30 1997-2004.

Gerdes LU, Gerdes C, Hansen PS, Klausen IC, Faergeman O \& Dyerberg J 1996 The apolipoprotein E polymorphism in Greenland Inuit in its global perspective. Human Genetics 98 546-550. (doi:10.1007/ s004390050257)

Glomset JA 1968 The plasma lecithins:cholesterol acyltransferase reaction. Journal of Lipid Research 9 155-167.

Goldstein JL \& Brown MS 1985 The LDL receptor and the regulation of cellular cholesterol metabolism. Journal of Cell Science. Supplement $\mathbf{3}$ 131-137. (doi:10.1242/jcs.1985.Supplement_3.13)

Gwynne JT \& Strauss JF III 1982 The role of lipoproteins in steroidogenesis and cholesterol metabolism in steroidogenic glands. Endocrine Reviews 3 299-329. (doi:10.1210/edrv-3-3-299)

Hayek T, Oiknine J, Brook JG \& Aviram M 1994 Role of HDL apolipoprotein $E$ in cellular cholesterol efflux: studies in apo $E$ knockout transgenic mice. Biochemical and Biophysical Research Communications 205 1072-1078. (doi:10.1006/bbrc.1994.2775)

Hirshfield AN 1991 Development of follicles in the mammalian ovary. International Review of Cytology 124 43-101.

Huang ZH, Gu D \& Mazzone T 2009 Role of adipocyte-derived apoE in modulating adipocyte size, lipid metabolism, and gene expression in vivo. American Journal of Physiology. Endocrinology and Metabolism 296 E1110-E1119. (doi:10.1152/ajpendo.90964.2008) 
Jo H, Choe SS, Shin KC, Jang H, Lee JH, Seong JK, Back SH \& Kim JB 2012 ER stress induces hepatic steatosis via increased expression of the hepatic VLDL receptor. Hepatology 57 1366-1377. (doi:10.1002/hep.26126)

Kaufman RJ, Scheuner D, Schroder M, Shen X, Lee K, Liu CY \& Arnold SM 2002 The unfolded protein response in nutrient sensing and differentiation. Nature Reviews. Molecular Cell Biology3 411-421. (doi:10.1038/nrm829)

Kockx M, Dinnes DL, Huang KY, Sharpe LJ, Jessup W, Brown AJ \& Kritharides L 2012 Cholesterol accumulation inhibits ER to Golgi transport and protein secretion: studies of apolipoprotein E and VSVGt. Biochemical Journal 447 51-60. (doi:10.1042/BJ20111891)

Lavoie HA \& King SR 2009 Transcriptional regulation of steroidogenic genes: STARD1, CYP11A1 and HSD3B. Experimental Biology and Medicine 234 880-907. (doi:10.3181/0903-MR-97)

Liu P, Ying Y, Zhao Y, Mundy DI, Zhu M \& Anderson RG 2004 Chinese hamster ovary K2 cell lipid droplets appear to be metabolic organelles involved in membrane traffic. Journal of Biological Chemistry 279 3787-3792. (doi:10.1074/jbc.M311945200)

Madrid LV, Mayo MW, Reuther JY \& Baldwin AS Jr 2001 Akt stimulates the transactivation potential of the RelA/p65 subunit of NF-KB through utilization of the $I \kappa B$ kinase and activation of the mitogen-activated protein kinase p38. Journal of Biological Chemistry 276 18934-18940. (doi:10.1074/jbc.M101103200)

Maehama T \& Dixon JE 1999 PTEN: a tumour suppressor that functions as a phospholipid phosphatase. Biological Chemistry 9 125-128.

Mahley RW 1988 Apolipoprotein E: cholesterol transport protein with expanding role in cell biology. Science 240 622-630. (doi:10.1126/ science.3283935)

Mahley RW \& Rall SC Jr 2000 Apolipoprotein E: far more than a lipid transport protein. Annual Review of Genomics and Human Genetics 1 507-537. (doi:10.1146/annurev.genom.1.1.507)

Margaret AL \& Dario RA 2001 PKB/Akt: a key mediator of cell proliferation, survival and insulin responses? Cell Science 114 2903-2910.

Meiner VL, Cases S, Myers HM, Sande ER, Bellosta S, Schambelan M, Pitas RE, McGuire J, Herz J \& Farese RV Jr 1996 Disruption of the acylCoA:cholesterol acyltransferase gene in mice: evidence suggesting multiple cholesterol esterification enzymes in mammals. PNAS 93 14041-14046. (doi:10.1073/pnas.93.24.14041)

Mendelson CR, Means GD, Mahendroo MS, Corbin CJ, Michael P, Steinkampf MP, Lorence SG \& Simpson ER 1990 Use of molecular probes to study regulation of aromatase cytochrome P-450. Biology of Reproduction 42 1-10. (doi:10.1095/biolreprod42.1.1)

Moreno JA, Lopez-Miranda J \& Perez-Jimenez F 2006 Influence of genetic and environmental factors on lipid metabolism and cardiovascular risk associated with the apoE gene. Medicina Clínica 127 343-351. (doi:10. 1157/13092316)

Ng DS, Francone OL, Forte TM, Zhang J, Haghpassand M \& Rubin EM 1997 Disruption of the murine lecithin:cholesterol acyltransferase gene causes impairment of adrenal lipid delivery and up-regulation of scavenger receptor class B type I. Journal of Biological Chemistry 272 15777-15781. (doi:10.1074/jbc.272.25.15777)

Nicosia M, Moger WH, Dyer CA, Prack MM \& Williams DL 1992 Apolipoprotein-E messenger RNA in rat ovary is expressed in theca and interstitial cells and presumptive macrophage, but not in granulosa cells. Molecular Endocrinology 6 978-988. (doi:10.1210/me.6.6.978)

Ogbuji QC 2010 Obesity and reproductive performance in women. African Journal of Reproductive Health 14 143-151.

Oyadomari S, Araki E \& Mori M 2002 Endoplasmic reticulum stress-mediated apoptosis in pancreatic $\beta$-cells. Apoptosis 7 335-345. (doi:10.1023/A:1016175429877)

Pfaffl MW 2001 A new mathematical model for relative quantification in realtime RT-PCR. Nucleic Acids Research 29 e45. (doi:10.1093/nar/29.9.e45)

Plump AS \& Breslow JL 1995 Apolipoprotein E and the apolipoprotein E-deficient mouse. Annual Review of Nutrition 15 495-518. (doi:10.1146/annurev.nu.15.070195.002431)

Plump AS, Smith JD, Hayek T, Aalto-Setala K, Walsh A, Verstuyft JG, Rubin EM \& Breslow JL 1992 Severe hypercholesterolemia and atherosclerosis in apolipoprotein E-deficient mice created by homologous recombination in ES cells. Cell 71 343-353. (doi:10.1016/00928674(92)90362-G)

Plump AS, Erickson SK, Weng W, Partin JS, Breslow JL \& Williams DL 1996 Apolipoprotein A-I is required for cholesteryl ester accumulation in steroidogenic cells and for normal adrenal steroid production. Journal of Clinical Investigation 97 2660-2671. (doi:10.1172/JCI118716)

Reddy P, Liu L, Adhikari D, Jagarlamudi K, Rajareddy S, Shen Y, Du C, Tang WL, Hämäläinen T, Peng SL et al. 2008 Oocyte-specific deletion of Pten causes premature activation of the primordial follicle pool. Science 319 611-613. (doi:10.1126/science.1152257)

Reyland ME \& Williams DL 1991 Suppression of cAMP-mediated signal transduction in mouse adrenocortical cells which express apolipoprotein E. Journal of Biological Chemistry 266 21099-21104.

Reyland ME, Gwynne JT, Forgez P, Prack MM \& Williams DL 1991 Expression of the human apolipoprotein $\mathrm{E}$ gene suppresses steroidogenesis in mouse Y1 adrenal cells. PNAS 88 2375-2379. (doi:10.1073/pnas. 88.6.2375)

Sasson R, Dantes A, Tajima K \& Amsterdam A 2003 Novel genes modulated by $\mathrm{FSH}$ in normal and immortalized FSH-responsive cells: new insights into the mechanism of FSH action. FASEB Journal 17 1256-1266. (doi:10.1096/fj.02-0740com)

Shore VG \& Shore B 1973 Heterogeneity of human plasma very low density lipoproteins. Separation of species differing in protein components. Biochemistry 12 502-507. (doi:10.1021/bi00727a022)

Tang X \& Zhang C 2011 Relationship between Sloan-Kettering virus expression and mouse follicular development. Endocrine 40 187-195. (doi:10.1007/s12020-011-9477-y)

Trigatti B, Rayburn H, Vinals M, Braun A, Miettinen H, Penman M, Hertz M, Schrenzel M, Amigo L, Rigotti A et al. 1999 Influence of the high density lipoprotein receptor SR-BI on reproductive and cardiovascular pathophysiology. PNAS 96 9322-9327. (doi:10.1073/pnas.96. 16.9322)

Wang XT, Pei DS, Xu J, Guan QH, Sun YF, Liu XM \& Zhang GY 2007 Opposing effects of Bad phosphorylation at two distinct sites by Akt1 and JNK1/2 on ischemic brain injury. Cell Signalling 19 1844-1856. (doi:10. 1016/j.cellsig.2007.04.005)

Weil S, Vendola K, Zhou J \& Bondy CA 1999 Androgen and folliclestimulating hormone interactions in primate ovarian follicle development. Journal of Clinical Endocrinology and Metabolism 84 2951-2956. (doi:10.1210/jc.84.8.2951)

Ye HY, Yin M, Shang YJ, Dai XD, Zhang SQ, Jing W, Du HQ, Zhang L \& Pan J 2008 Differential expressions of lipid metabolism related genes in the liver of young apoE knockout mice. Sheng Li Xue Bao 60 51-58.

Yue L \& Spradling AC 1992 hu-li tai shao, a gene required for ring canal formation during Drosophila oogenesis, encodes a homolog of adducin. Genes and Development 6 2443-2454. (doi:10.1101/gad.6.12b.2443)

Zerbinatti CV, Mayer LP, Audet RG \& Dyer CA 2001 Apolipoprotein E is a putative autocrine regulator of the rat ovarian theca cell compartment. Biology of Reproduction 64 1080-1089. (doi:10.1095/biolreprod64.4. 1080)

Zhang SH, Reddick RL, Piedrahita JA \& Maeda N 1992 Spontaneous hypercholesterolemia and arterial lesions in mice lacking apolipoprotein E. Science 258 468-471. (doi:10.1126/science.1411543)

Zofkova I, Zajickova K, Hill M \& Horinek A 2002 Apolipoprotein E gene determines serum testosterone and dehydroepiandrosterone levels in postmenopausal women. European Journal of Endocrinology 147 503-506. (doi:10.1530/eje.0.1470503)

Received 9 February 2013

First decision 25 March 2013

Revised manuscript received 22 September 2013

Accepted 4 November 2013 